\title{
Modification of Lysine Residues to Alkyl-substituted Pyridiniums on Exposure of Proteins to Vaporized Hexanal
}

\author{
Hiromichi Kato, Shigenobu KaneKo, Akihiro OKitani, ${ }^{*}$ \\ Masayo ITO and Fumitaka HAYASE \\ Department of Agricultural Chemistry, The University of Tokyo, \\ Bunkyo-ku, Tokyo 113, Japan \\ *Department of Food Science and Technology, Nippon Veterinary and Zootechnical College, \\ Musashino-shi, Tokyo 180, Japan
}

Received November 7, 1985

\begin{abstract}
We examined the structure of lysine residues which had been impaired due to exposure of proteins to hexanal, using a free lysine/hexanal system (I), a Gly-His-Lys/hexanal system (II) and a lysozyme/hexanal system (III). The most abundant ninhydrin positive compound (N-50) was isolated from the products with system I and identified as 1-(5-carboxy-5-aminopentyl)-2-pentyl3,5-dibutylpyridinium betaine, which was form ${ }^{\circ}$ d through condensation of $\varepsilon$-amino groups of lysine with three molecules of hexanal.

A new ninhydrin positive compound ( $\mathrm{N}-51$ ) was isolated from hydrolysates of the products with systems II and III, and identified as the same compound as N-50 from the UV and mass spectra and thin layer chromatogram.

These results indicated that the $\varepsilon$-amino groups of lysine residues of proteins are modified to alkyl-substituted pyridinium rings on exposure to hexanal.
\end{abstract}

For oxidizing lipids, hydroperoxides are formed in the first step and then carbonyl compounds such as aldehydes and ketones are formed through secondary degradation of the hydroperoxides. These oxidized lipids cause various forms of deterioration of proteins such as browning, polymerization and decreased solubility. ${ }^{1 \sim 7)}$ These changes in proteins are usually accompanied by impairment of certain amino acid residues. The labile amino acid residues on reaction with oxidized lipids were reported to be methionine, lysine, histidine, tryptophan and tyrosine residues. ${ }^{1,4,6,8,9)}$ However, the relationship of the impairment of these amino acid residues to the above-mentioned forms of deterioration of proteins is almost completely unknown.

Among these amino acid residues, lysine residues seem to cause the deterioration of proteins through an amino-carbonyl reaction with secondary products from lipid oxidation. Shimada and Matsushita ${ }^{10)}$ reported that the polymerization of soybean $11 \mathrm{~S}$ globulin due to hydroperoxy-linoleic acid was retarded by masking of amino groups of the protein by means of acetylation or succinylation. We reported that the polymerization and browning of lysozymes exposed to vaporized hexanal, which is one of the principal secondary products on oxidation of linoleic acid, were retarded by masking of amino groups of the proteins. ${ }^{11)}$ These results suggested that the reaction involving lysine residues was responsible for the cross-linking of proteins. Thus, in the present study we investigated the structure of the impaired lysine residues of lysozymes exposed to hexanal vapor.

\section{MATERIALS AND METHODS}

Materials. Lysozyme (egg white, $6 \times$ crystallized) was purchased from Seikagaku Kogyo, Tokyo. Lysine (free base) and lysine hydrochloride were from Nakarai Chemicals, Kyoto, and Kanto Chemicals, Tokyo, respectively. Gly-L-His-L-Lys was from Sigma. n-Hexanal was from Tokyo Kasei Chemicals, Tokyo. 
Reaction systems. The lysozyme/hexanal system reaction was performed with the protein in the solid state as described previously. ${ }^{11)}$ Lyophilized lysozymes $(100 \mathrm{mg})$ were wrapped in tissue paper and stored over $3 \mathrm{ml}$ hexanal at $50^{\circ} \mathrm{C}$ and $75 \%$ relative humidity $(\mathrm{RH})$ for 5 days in a $500 \mathrm{ml}$ cell. After storage, the lysozymes were washed with an acetone-methanol mixture $(1: 1, \mathrm{v} / \mathrm{v})$ and then with ether. The washed lysozymes were then subjected to analysis.

The lysine/hexanal system reaction was performed in a solid state system or an aqueous system. In the solid state system, free base lysine powder was stored as in the case of the lysozyme/hexanal system, except that the lysine powder was stored on a petri-dish because it absorbs water and dissolves at $75 \% \mathrm{RH}$. When a large amount of the reaction product was needed, lysine was reacted with hexanal in an aqueous system as described below. Lysine hydrochloride was dissolved in water, and then the $\mathrm{pH}$ of the solution was adjusted to 6.8 with sodium hydrogen carbonate. A one-seventh volume of methanol was added to the solution to increase the solubility of hexanal. After addition of an equimolar amount of hexanal to the lysine, the reaction mixture was refluxed at $50^{\circ} \mathrm{C}$ for $7 \mathrm{hr}$ and then stirred at room temperature for $24 \mathrm{hr}$.

Gly-His-Lys was reacted in the solid state with hexanal as in the case of the lysine/hexanal system.

Thin layer chromatography for analysis of modified lysine residues. Thin layer chromatographies (TLC) were performed for analysis of lysine and modified lysine residues of lysozymes. Merck 5735 plastic sheets containing a fluorescent reagent, that is excited at $254 \mathrm{~nm}$, or Whatman high performance thin layer plates, LHP-K, were used for silica gel TLC, and Funakoshi Avicel SF was used for cellulose TLC. $n$-Butanol-acetic acid-water $(4: 1: 1$ or $4: 1: 2$ ) was used as the developing solution. Spots were detected after spraying ninhydrin solution $(0.2 \%$ in ethanol), and from the fluorescence on excitation at $365 \mathrm{~nm}$ and the UV absorption at $254 \mathrm{~nm}$.

Chromatography. The reaction products were purified by paper partition chromatography (PPC), preparative TLC and silica gel column chromatography. Toyo 51A or 514A filter papers were used for PPC and Merck 5745 silica gel plates for preparative TLC. Silica gel column chromatography was performed with $100 \sim 200$ mesh silica gel. The developing solution for the above chromatographies was $n$-butanol-acetic acid-water ( $4: 1: 2$ for PPC and TLC, and $4: 1: 1$ for the column chromatography). The developed samples were extracted from the filter papers or the thin layer plates with methanol. The purity of the samples was checked by TLC as described in the previous section.

Spectrometry. UV absorption was monitored with a Hitachi Model 100-60 spectrophotometer. Mass spectra were recorded with a JEOL DX-300 mass spectrometer.
The ionization modes were EI, CI (with isobutane as the reaction gas) and FD (emitter, silicon emitter; cathode voltage, $5.5 \mathrm{kV}$ ). The ${ }^{13} \mathrm{C}-\mathrm{NMR}$ spectra were recorded with a JEOL-FX-100 NMR spectrometer operated at $25 \mathrm{MHz}$ in the pulse Fourier transition mode. This spectrum was recorded in $\mathrm{D}_{2} \mathrm{O}$ using 8,000 data points and a spectral width of $6 \mathrm{kHz} .{ }^{13} \mathrm{C}$-Chemical shifts are expressed with methanol as an internal standard. ${ }^{1} \mathrm{H}-\mathrm{NMR}$ spectra were recorded with a JNM-4H-100 spectrometer in $\mathrm{D}_{2} \mathrm{O}$.

\section{RESULTS}

I. The structure of the reaction product from free lysine and hexanal

The reaction mixture obtained after exposing lysine powder to hexanal was developed on a silica gel thin layer with a solvent system of butanol-acetic acid-water $(4: 1: 1)$. As shown in Fig. 1, the chromatogram showed 10 ninhydrin positive compounds $(\mathrm{N}-10 \sim \mathrm{N}$ 100) as well as unreacted lysine. Some spots of fluorescent products and UV-absorbing products other than $\mathrm{N}-10 \sim \mathrm{N}-100$ were also observed. In the present study, we investigated the ninhydrin positive compounds further, because the impaired lysine residues in lysozymes might have intact $\alpha$-amino

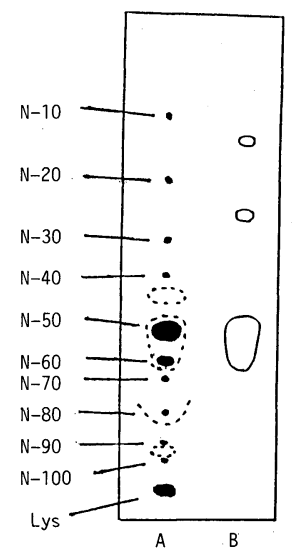

FIG. 1. Silica Gel Thin Layer Chromatography of the Lysines Modified on Exposure of Free Lysines to Vaporized Hexanal at $50^{\circ} \mathrm{C}$ and $75 \%$ RH for 2 Days.

The modified lysines were dissolved in methanol and developed with butanol-acetic acid-water $(4: 1: 1)$. The spots were detected with ninhydrin (A) and UV (254 nm) irradiation (B). The fluorescence seen on excitation at $365 \mathrm{~nm}$ is shown by dotted lines (A). Ninhydrin positive compounds were numbered $\mathrm{N}-10 \sim \mathrm{N}-100$, as shown in the figure. 


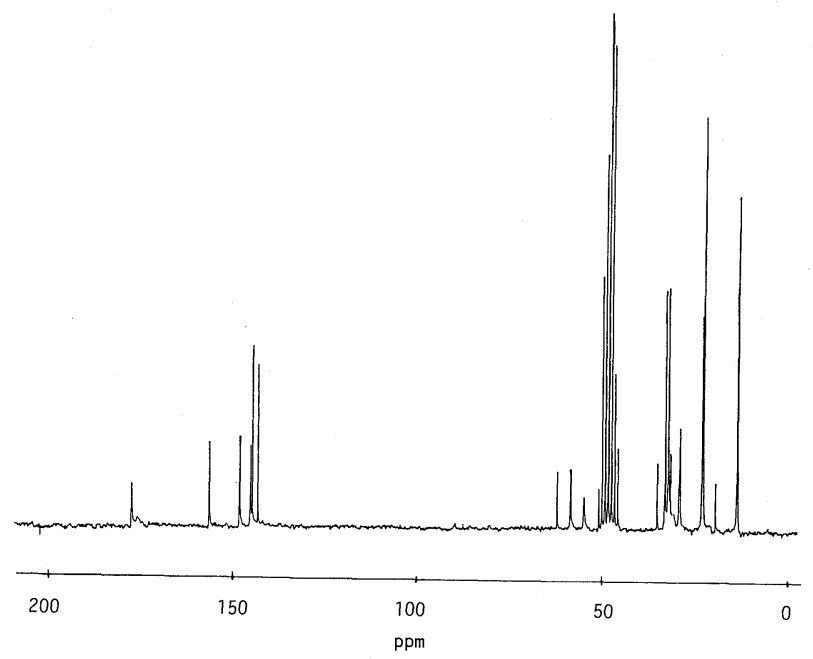

Fig. 2. ${ }^{13} \mathrm{C}-\mathrm{NMR}$ Spectrum of $\mathrm{N}-50$.

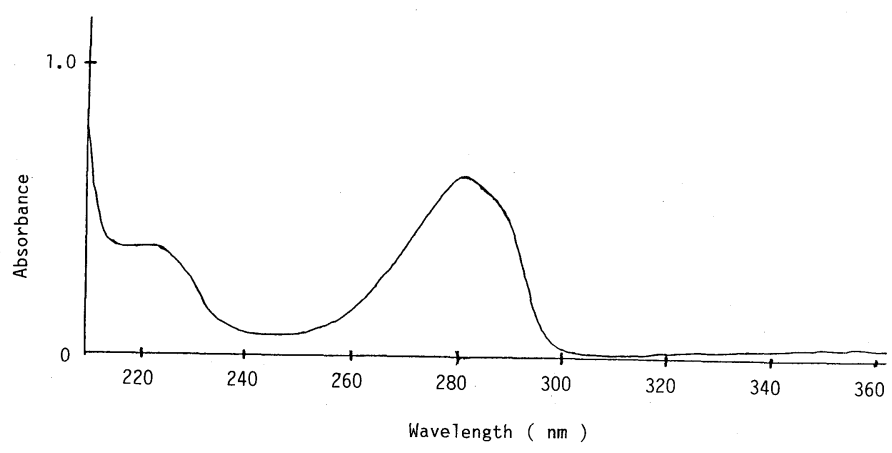

FIG. 3. UV Spectrum of N-50.

groups that are detectable with ninhydrin after $\mathrm{HCl}$-hydrolysis. $\mathrm{N}-50$ was the most abundant product with this system and $\mathrm{N}-60$ the next most abundant. Other ninhydrin positive compounds were produced in small quantities compared with $\mathrm{N}-50$ and $\mathrm{N}-60$.

Isolation of the most abundant product, $\mathrm{N}$ 50 , from the reaction mixture was performed as follows. The reaction mixture of the aqueous system described under MATERIALS AND Methods was washed with water to remove unreacted lysine and then with ether to remove unreacted hexanal and other ether-soluble compounds. Ninhydrin positive compounds with $R f$ values higher than that of $\mathrm{N}-50$ were also removed through a wash with ether. N-50 was partitioned with water and ether in equal proportions through these procedures. Subse- sequently, $\mathrm{N}-50$ was purified by $\mathrm{PPC}$, which removed ninhydrin positive compounds with $R f$ values lower than that of N-50. Finally, preparative TLC and silica gel column chromatography were performed successively to remove ninhydrin-negative, fluorescent compounds.

${ }^{1} \mathrm{H}-\mathrm{NMR},{ }^{13} \mathrm{C}-\mathrm{NMR}$ (Fig. 2), mass and UV (Fig. 3) measurements of $\mathrm{N}-50$ gave the following data: $\mathrm{UV} \lambda_{\max }^{\mathrm{H}_{2} \mathrm{O}} \mathrm{nm}$ : 223, 281 (inflection $289 \mathrm{~nm}$ ). EI-milli MS $m / z$ : 261.24427 $\left(\mathrm{C}_{18} \mathrm{H}_{31} \mathrm{~N}_{1}\right)$ CI-MS $m / z$ : 262. FD-MS $m / z$ : $391\left(\mathrm{M}+\mathrm{H}^{+}\right) .{ }^{1} \mathrm{H}-\mathrm{NMR} \quad \delta: 8.40(1 \mathrm{H}, \mathrm{s})$, $7.92(1 \mathrm{H}, \mathrm{s}), 3.40(1 \mathrm{H}, \mathrm{t}), 2.6 \sim 3.0(8 \mathrm{H}, \mathrm{m})$, $1.0 \sim 1.6(20 \mathrm{H}, \mathrm{m}), 0.6 \sim 0.8(9 \mathrm{H}, \mathrm{m}$ of $\mathrm{t}) .{ }^{13} \mathrm{C}-$ NMR $\delta: 176.8$ (s), 155.9 (s), 147.8 (d), 144.9 (d), $144.4(\mathrm{~s}), 142.9$ (s), 59.9 (t), 56.4 (d), 34.4 $(2 \mathrm{C}, \mathrm{t}), 33.5(2 \mathrm{C}, \mathrm{t}), 33.0(\mathrm{t}), 30.5(\mathrm{t}), 30.3(\mathrm{t})$, $24.0(2 \mathrm{C}, \mathrm{t}), 22.7(4 \mathrm{C}, \mathrm{t}), 15.2(\mathrm{q}), 15.0(2 \mathrm{C}, \mathrm{q})$. 


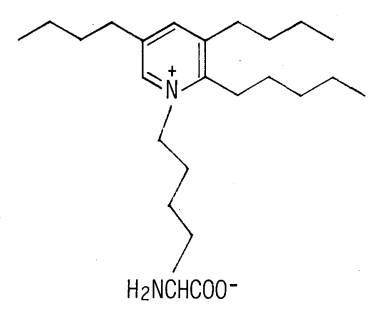

FIG. 4. Structure of N-50, 1-(5-Carboxy-5-aminopentyl)-2-pentyl-3,5-dibutylpyridinium Betaine.

The mass data indicate that the molecular weight of $\mathrm{N}-50$ is 390 and that it contains a stable partial structure with a molecular weight of 261 . Both the ${ }^{1} \mathrm{H}-\mathrm{NMR}$ and ${ }^{13} \mathrm{C}$ NMR spectra show three methyl groups ascribable to three molecules of hexanal. The ${ }^{1} \mathrm{H}-\mathrm{NMR}$ spectrum shows heteroaromatic protons at $8.4 \mathrm{ppm}$ and $7.9 \mathrm{ppm}$. In addition, the UV spectrum of N-50 was similar to those of isodesmosine ${ }^{12)}$ and the alkyl-substituted pyridinium betaines synthesized by Suyama and Adachi. ${ }^{13,14)}$ These spectral data indicate that N-50 has an alkyl-substituted pyridinium ring which is formed through the condensation of an $\alpha$-or $\varepsilon$-amino group of lysine and three molecules of hexanal.

Comparison of the ${ }^{13} \mathrm{C}-\mathrm{NMR}$ data of N-50 with those of lysine $\left(\alpha\right.$-carbon; $55.3 \mathrm{ppm}: \varepsilon^{-}$ carbon; $40.0 \mathrm{ppm})^{15)}$ revealed the the $\varepsilon$-carbongenerated signal of N-50 was shifted to a low magnetic field (59.9 ppm) while the $\alpha$-carbongenerated signal was not shifted $(56.4 \mathrm{ppm})$. These results revealed that $\mathrm{N}-50$ is formed through the condensation of an $\varepsilon$-amino group of lysine. Thus, N-50 was identified as 1-(5carboxy-5-aminopentyl)-2-pentyl-3,5-dibutylpyridinium betaine (Fig. 4).

\section{The structure of the reaction product from} lysine residues of proteins and hexanal

On the basis of the data obtained for the reaction product from the above-mentioned free lysine/hexanal system, the structure of modified lysine residues in lysozymes exposed to vaporized hexanal was analyzed.

Exposure to hexanal for 5 days resulted in impairment of about $90 \%$ of the lysine residues of lysozymes. These hexanal-treated lysozymes

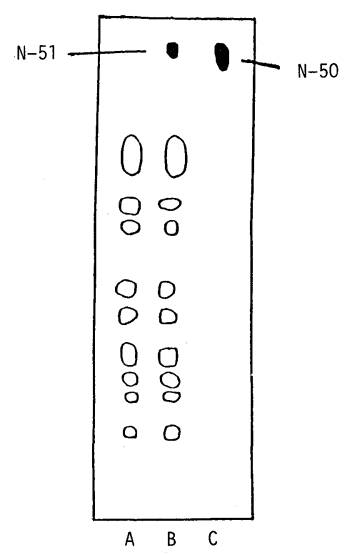

FIG. 5. Cellulose Thin Layer Chromatography of Hydrolysates of Lysozymes Exposed to Hexanal Vapor.

Lysozymes before (A) and after (B) exposure to vaporized hexanal were hydrolyzed for $48 \mathrm{hr}$ with $6 \mathrm{~N} \mathrm{HCl}$. The hydrolysates and N-50 (C) were developed with butanol-acetic acid-water $(4: 1: 2)$. Spots were detected with ninhydrin. $\bigcirc$, violet spots; $\bigcirc$, blue spots.

were hydrolyzed with $6 \mathrm{~N} \mathrm{HCl}$ at $110^{\circ} \mathrm{C}$ for $48 \mathrm{hr}$, and then the hydrolysates were subjected to cellulose thin layer chromatography with a solvent system of butanol-acetic acid-water (4:1:2). As shown in Fig. 5, the chromatogram showed a new ninhydrin positive compound (N-51) with a markedly high $R f(0.91)$ as well as common amino acids contained in lysozymes. On a cellulose plate, N-51 was stained blue by ninhydrin reagent while other amino acids were stained violet.

Purification of N-51 from the hydrolysates was conducted by successive PPC, TLC and silica gel column chromatography with butanol-acetic acid-water $(4: 1: 2)$ as the solvent system. N-51 could be readily separated and distinguished from the other amino acids on PPC because of its high $R f$ and ninhydrindeveloped blue color. The TLC and silica gel column chromatography separated N-51 from UV absorbing compounds which were formed during the hydrolysis with $6 \mathrm{~N} \mathrm{HCl} . \mathrm{N}-51$ behaved exactly the same as $\mathrm{N}-50$ did on thin layer chromatography on both a silica gel plate and a cellulose plate (Fig. 5). Measurement of the UV and mass spectra of $\mathrm{N}-51$ gave the following data: UV $\lambda_{\max }^{\mathrm{H}_{2} \mathrm{O}} \mathrm{nm}: 223,281$ 


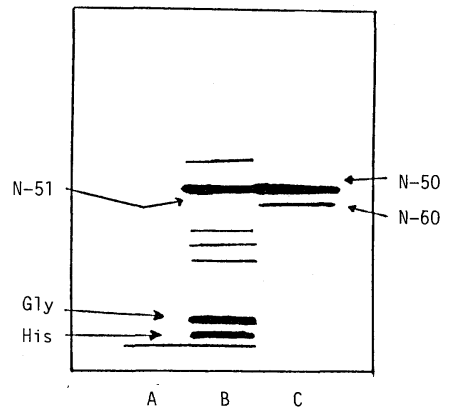

FIG. 6. High Performance Thin Layer Chromatography of Hydrolysates of Gly-His-Lys Exposed to Hexanal Vapor at $50^{\circ} \mathrm{C}$ and $75 \% \mathrm{RH}$ for $21 \mathrm{hr}$.

Lysine (A), Gly-His-Lys after exposure to hexanal (B) and a mixture of $\mathrm{N}-50$ and $\mathrm{N}-60$ (C) were developed with butanol-acetic acid-water $(4: 1: 2)$ on a Whatman LHP-K silica gel plate. Spots were detected with ninhydrin.

(inflection $289 \mathrm{~nm}$ ). EI-MS $m / z: 261.24124$ $\left(\mathrm{C}_{18} \mathrm{H}_{31} \mathrm{~N}_{1}\right)$. CI-MS $m / z$ : 262. FD-MS $m / z$ : 391 $\left(\mathrm{M}+\mathrm{H}^{+}\right)$. These data were identical to those for N-50. These results showed that N-51 and $\mathrm{N}-50$ are the same compound, 1-(5-carboxy-5aminopentyl)-2-pentyl-3,5-dibutylpyridinium betaine.

A tripeptide, Gly-His-Lys, was reacted with hexanal and the reaction product was compared with that of the free lysine/hexanal system. After Gly-His-Lys that had been exposed to hexanal vapor was hydrolyzed with $6 \mathrm{~N} \mathrm{HCl}$ at $110^{\circ} \mathrm{C}$ for $24 \mathrm{hr}$, the hydrolysates were subjected to high performance thin layer chromatography together with $\mathrm{N}-50$ and N-60 with a solvent system of butanol-acetic acidwater $(4: 1: 2)$. The results shown in Fig. 6 indicate that the Gly-His-Lys/hexanal system produced $\mathrm{N}-50$ but not $\mathrm{N}-60$. This result showed that $\mathrm{N}-60$, one of the principal products with the free lysine/hexanal system, was not generated from a peptide that had reacted with hexanal.

\section{DISCUSSION}

The $\varepsilon$-amino groups of lysine residues of proteins were found to be modified to alkylsubstituted pyridinium rings on exposure to vaporized hexanal. N-50, i.e., 1-(5-carboxy-5-

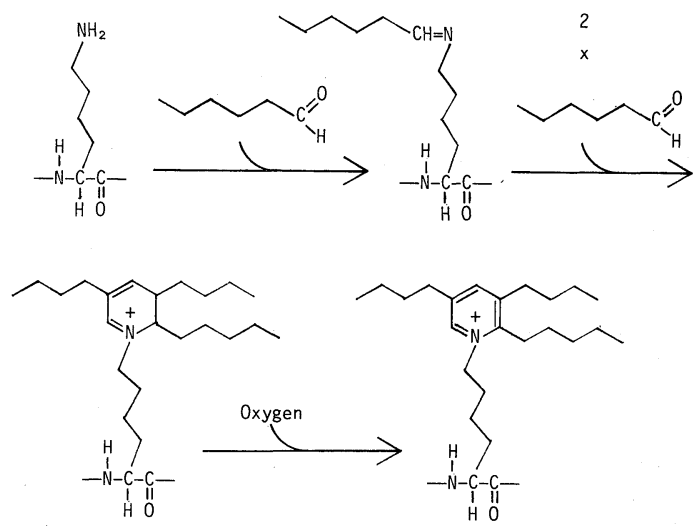

FIG. 7. Proposed Scheme for the Production of N-50 from Lysine Residues and Hexanal.

aminopentyl)-2-pentyl-3,5-dibutylpyridinium betaine, was the identified reaction product. Suyama and Adachi ${ }^{13)}$ reported that reactions between various primary amines and aliphatic aldehydes in aqueous systems produce the respective pyridinium betaines, which are analogues of N-50. Our results show that their reaction mechanism is applicable to the hexanalmodified lysine residues in proteins. Suyama and Adachi $^{13}$ ) proposed that the process of the formation of a pyridinium ring involved Schiff-base formation as the first step and subsequent aldol condensation. The last step in the formation of the pyridinium rings is assumed to require oxygen or another oxidizing agent ${ }^{13)}$ (Fig. 7). In our previous paper, ${ }^{11)}$ we reported that the various forms of deterioration of lysozymes including impairment of lysine residues are retarded when oxygen is removed from the reaction cell. As regards the impairment of lysine residues, oxygen may be required as an oxidizing agent for formation of the pyridinium ring.

$\mathrm{N}-60$, the second most abundant product with the lysine/hexanal system, was not detected with the Gly-His-Lys/hexanal system. This result shows that N-60 is not formed when $\alpha$-amino groups of lysine are masked by peptide bonds. Thus, N-60 is assumed to be formed through the reaction between an $\alpha$ amino group and hexanal. The mass spectra of $\mathrm{N}-60$, that had been separated by high perform- 
ance thin layer chromatography, was found to be identical to those of N-50. These results are explained by the assumption that N-60 is formed through modification of an $\alpha$-amino group to give the same pyridinium ring as that of N-50. The formation of N-60 is not expected for lysine residues in inner parts of a polypeptide chain. On the other hand, modification of $\alpha$-amino groups may occur in the case of $\mathrm{N}$-terminal lysine residues of lysozymes. However, we did not investigate the occurrence of N-60 in hexanal-modified lysozymes in the present study.

On exposure to hexanal, lysozymes suffer various forms of deterioration such as browning, polymerization and decreased solubility. ${ }^{11)}$ As shown by the cellulose thin layer chromatogram in Fig. 5, N-50 is a much more hydrophobic amino acid derivative than other common amino acids. The increase in hydrophobicity due to the formation of N-50 is assumed to be one of the causes of the reduction in solubility of lysozymes. However, there must be other causes for the hexanalinduced polymerization and discolorization of proteins because these alkyl-substituted pyridiniums derived from lysine residues should be neither cross-linkers of proteins nor brown pigments. Considering the previous findings ${ }^{11)}$ that browning and polymerization of lysozymes due to hexanal are retarded by acetylation of lysine residues, it is suggested that all of the lysine residues of lysozymes are not converted to $\mathrm{N}-50$, but that some of them are converted to the compounds responsible for the browning and polymerization. We are now studying these compounds.

\section{REFERENCES}

1) W. T. Roubal and A. L. Tappel, Arch. Biochem. Biophys., 113, 5 (1966).

2) P. T. Gamage, T. Mori and S. Matsushita, J. Nutr. Sci. Vitaminol., 19, 173 (1973).

3) J. Kanner and M. Karel, J. Agric. Food Chem., 24, 468 (1976).

4) H. W. Gardner, J. Agric. Food Chem., 27, 220 (1979).

5) L. Leake and M. Karel, J. Food Sci., 47, 737 (1982).

6) T. Matoba, H. Yoshida and D. Yonezawa, Agric. Biol. Chem., 46, 979 (1982).

7) J. Funes, S. Yong and M. Karel, J. Agric. Food Chem., 28, 794 (1980).

8) K. Kanazawa, G. Danno and M. Natake, J. Nutr. Sci. Vitaminol., 21, 373 (1975).

9) T. Matoba, D. Yonezawa, B. M. Nair and M. Kito, J. Food Sci., 49, 1082 (1984).

10) K. Shimada and S. Matsushita, Agric. Biol. Chem., 42, 781 (1978).

11) Y. Tashiro, A. Okitani, N. Utsunomiya, S. Kaneko and H. Kato, Agric. Biol. Chem., 49, 1739 (1985).

12) J. Thomas, D. F. Elsden and S. M. Partridge, Nature, 200, 651 (1963).

13) K. Suyama and S. Adachi, J. Org. Chem., 44, 1417 (1979).

14) T. Nakanishi and K. Suyama, Agric. Biol. Chem., 38, 1141 (1974)

15) E. Breitmaier, G. Haas and W. Voelter, "Atlas of Carbon-13 NMR Data," Heiden \& Sons, 1979. 